\title{
ON MATRICES WHOSE REAL LINEAR COMBINATIONS ARE NONSINGULAR
}

\author{
J. F. ADAMS, PETER D. LAX ${ }^{1}$ AND RALPH S. PHILLIPS ${ }^{2}$
}

Let $\Lambda$ be either the real field $R$, or the complex field $C$, or the skew field $Q$ of quaternions. Let $A_{1}, A_{2}, \cdots, A_{k}$ be $n \times n$ matrices with entries from $\Lambda$. Consider a typical linear combination $\sum_{j=1}^{n} \lambda_{j} A_{j}$ with real coefficients $\lambda_{j}$; we shall say that the set $\left\{A_{j}\right\}$ "has the property $P$ " if such a linear combination is nonsingular (invertible) except when all the coefficients $\lambda_{j}$ are zero.

We shall write $\Lambda(n)$ for the maximum number of such matrices which form a set with the property $P$. We shall write $\Lambda_{H}(n)$ for the maximum number of Hermitian matrices which form a set with the property $P$. (Here, if $\Lambda=R$, the word "Hermitian" merely means "symmetric"; if $\Lambda=Q$ it is defined using the usual conjugation in $Q$.) Our aim is to determine the numbers $\Lambda(n), \Lambda_{H}(n)$.

Of course, it is possible to word the problem more invariantly. Let $W$ be a set of matrices which is a vector space of dimension $k$ over $R$; we will say that $W$ "has the property $P$ " if every nonzero $w$ in $W$ is nonsingular (invertible). We now ask for the maximum possible dimension of such a space.

In [1], the first named author has proved that $R(n)$ equals the socalled Radon-Hurwitz function, defined below. In this note we determine $R_{H}(n), C(n), C_{H}(n), Q(n)$ and $Q_{H}(n)$ by deriving inequalities between them and $R(n)$. The elementary constructions needed to prove these inequalities can also be used to give a simplified description of the Radon-Hurwitz matrices.

The study of sets of real symmetric matrices $\left\{A_{j}\right\}$ with the property $P$ may be motivated as follows. For such a set, the system of partial differential equations

$$
u_{t}=\sum_{j} A_{j} u_{x_{j}}
$$

is a symmetric hyperbolic system in which the sound speeds are nonzero in every direction. For such systems the solution energy is propagated to infinity and a scattering theory can be developed.

To give our results, we require the Radon-Hurwitz numbers [2],

Received by the editors September 10, 1963.

1 Sloan Fellow.

2 Sponsored by the National Science Foundation, contract NSF-G 16434. 
[3]. We set $n=(2 a+1) 2^{b}$ and $b=c+4 d$, where $a, b, c, d$ are integers with $0 \leqq c<4$; then we define

$$
\rho(n)=2^{c}+8 d .
$$

ThEOREM 1. We have

$$
\begin{array}{ll}
R(n)=\rho(n), & R_{H}(n)=\rho\left(\frac{1}{2} n\right)+1, \\
C(n)=2 b+2, & C_{H}(n)=2 b+1, \\
Q(n)=2 b+4, & Q_{H}(n)=2 b+1 .
\end{array}
$$

The results for $\Lambda=Q$ are included so that topologists may avoid jumping to the conclusion that the subject is directly related to the Bott periodicity theorems. If this were so then it would be surprising to see the case $\Lambda=Q$ behaving like the case $\Lambda=C$.

The proof of Theorem 1 will be based on a number of simple constructions, which we record as lemmas.

Lemma 1. $R_{H}(n) \leqq C_{H}(n) \leqq Q_{H}(n)$.

This is clear, since a matrix with entries from $R$ may be regarded as a matrix with entries from $C$, and similarly for $C$ and $Q$.

Lemma 2. (a) $C(n) \leqq R(2 n)$, (b) $Q(n) \leqq C(2 n)$.

Proof. We may regard our matrices as $\Lambda$-linear transformations of coordinate $n$-space $\Lambda^{n}$. Now by forgetting part of the structure of $C^{n}$ it becomes a real vector space of dimension $2 n$ over $R$, i.e., an $R^{2 n}$. Thus any $C$-linear transformation of $C^{n}$ gives an $R$-linear transformation of $R^{2 n}$. Similarly for $C$ and $Q$.

Lemma 3. $\Lambda(n)+1 \leqq \Lambda_{H}(2 n)$.

PRoof. Let $W$ be a $k$-dimensional space of $n \times n$ matrices with entries from $\Lambda$ which has the property $P$. For each $A \in W$ and $\lambda \in R$, consider the following linear transformation from $\Lambda^{n} \oplus \Lambda^{n}$ to itself.

$$
B(x, y)=\left(A y+\lambda x, A^{*} x-\lambda y\right) .
$$

It is clear that its matrix is Hermitian, and that such $B$ form a $(k+1)$-dimensional space. We claim that this set $\{B\}$ has property $P$. For suppose that some $B$ is singular; then there exist $x, y$ not both zero such that

$$
A y+\lambda x=0, \quad A^{*} x-\lambda y=0 .
$$

Evaluating $x^{*} A y$ in two ways, we find 


$$
\lambda\left(x^{*} x+y^{*} y\right)=0 .
$$

Hence $\lambda=0$. Thus either $A$ or $A^{*}$ is singular; so $A$ is singular and $A=0$. This proves the lemma.

$$
\text { LEMma 4. (a) } C_{H}(n)+1 \leqq C(n) \text {, (b) } Q_{H}(n)+3 \leqq Q(n) \text {. }
$$

Proof. Let $W$ be a $k$-dimensional space of $n \times n$ Hermitian matrices with entries from $\Lambda$ which has the property $P$. Consider the matrices

$$
A+\mu I
$$

where $A$ runs over $W$ and $\mu$ runs over the pure imaginary elements of $\Lambda$. We claim that they form a space with the property $P$ and of dimension $k+1$ if $\Lambda=C$ or $k+3$ if $\Lambda=Q$. In fact, suppose that such a matrix is singular; then there is a nonzero $x$ such that

$$
A x=-\mu x ;
$$

arguing as is usual for the complex case, we find

$$
-\mu x^{*} x=x^{*} A x=(-\mu x)^{*} x=\mu x^{*} x .
$$

So $\mu$ is zero, $A$ is singular, and thus $A$ is zero. This proves the lemma.

LEMMA 5. $R_{H}(n)+7 \leqq R(8 n)$.

Proof. Let $W$ be a $k$-dimensional space of real symmetric matrices with the property $P$. We require also the Cayley numbers $K$, which form an 8-dimensional algebra over $R$. We can thus form the real vector space

$$
R^{n} \otimes_{R} K
$$

of dimension $8 n$. For each $A \in W$ and each pure imaginary $\mu \in K$ we consider the following linear transformation from $R^{n} \otimes_{R} K$ to itself:

$$
B(x \otimes y)=A x \otimes y+x \otimes \mu y .
$$

We claim that the $(k+7)$-dimensional space formed by such $B$ has property $P$. For suppose that some $B$ is singular, and suppose, to begin with, that $\mu$ is nonzero. Then the elements $1, \mu$ form an $R$-base for a sub-algebra of $K$ which we may identify with $C$. Now every two elements of $K$ generate an associative sub-algebra; in particular, $K$ is a left vector space over $C$. Choose a $C$-base of $K$; this splits $R^{n} \otimes_{R} K$ as the direct sum of 4 copies of $R^{n} \otimes_{R} C$. Since $B$ acts on each summand, it must be singular on at least one. That is, the real symmetric 
matrix $A$ has a nonzero complex eigenvalue which is purely imaginary-a contradiction. Hence $\mu$ must be zero and $B=A \otimes 1$. Now choose an $R$-base of $K$; this splits $R^{n} \otimes_{R} K$ as the direct sum of 8 copies of $R^{n}$. Since $B$ acts on each summand, it must be singular on at least one. That is, $A$ must be singular; hence $A=0$. This completes the proof.

Proof of Theorem 1. First we consider $R_{H}(n)$. If we use the fact that $R(n)=\rho(n)$, Lemmas 3 and 5 give

$$
\rho\left(\frac{1}{2} n\right)+1 \leqq R_{H}(n) \leqq \rho(8 n)-7 .
$$

But using the explicit definition of $\rho$, we have

$$
\rho(8 n)-7=\rho\left(\frac{1}{2} n\right)+1 .
$$

This disposes of $R_{H}(n)$.

It follows from this argument that if we have a set of $\rho(n) n \times n$ matrices with the property $P$, then by applying successively the constructions given in the proofs of Lemma 3 (taking $\Lambda=R$ ) and Lemma 5, we obtain a set of $\rho\left(2^{4} n\right) 2^{4} n \times 2^{4} n$ matrices with the property $P$. Now the set of $1,2,4$, and 8 matrices which express the respective actions of $R, C, Q$, and $K$ on $R^{m}, C^{m}, Q^{m}$, and $K^{m}$ for $m=2 a+1$ can be used to start the induction for the different cases $b \equiv 0,1,2$, and $3(\bmod 4)$. This gives a slight variation of the construction of Hurwitz and Radon [2], [3]; the iterative procedures used by these authors require more steps and do not involve the Cayley numbers explicitly.

Next we consider $C(n)$. Lemmas 3 and 4 (a) give

$$
C(n)+2 \leqq C(2 n) \text {. }
$$

Now induction shows that

$$
C(n) \geqq 2 b+2,
$$

which gives us our inequality one way. Applying Lemma 2(a) directly only gives a good inequality for certain values of $b$, so we proceed as follows. Choose $e \geqq 0$ such that $b+e \equiv 0$ or $1(\bmod 4)$. Then by induction we have

$$
C(n)+2 e \leqq C\left(2^{e} n\right)
$$

by Lemma $2(a)$ we have

$$
C\left(2^{e} n\right) \leqq \rho\left(2^{e+1} n\right),
$$

and by our choice of $e$ we have 


$$
\rho\left(2^{e+1} n\right)=2 b+2 e+2 .
$$

This gives

$$
C(n) \leqq 2 b+2,
$$

and proves the assertion made about $C(n)$.

Lemmas 3 and 4(a) now show that

$$
C_{H}(n)=2 b+1 \text {. }
$$

Again, Lemmas 1, 4(b) and 2(b) show that

$$
\begin{aligned}
Q_{H}(n)-C_{H}(n) & \geqq 0, \\
Q(n)-Q_{H}(n) & \geqq 3, \\
C(2 n)-Q(n) & \geqq 0 .
\end{aligned}
$$

But $C(2 n)-C_{H}(n)=3$, so all these inequalities are equalities. This completes the proof of Theorem 1 .

\section{REFERENCES}

1. J. F. Adams, Vector fields on spheres, Ann. of Math. (2) 75 (1962), 603-632.

2. A. Hurwitz, Über die Komposition der quadratischen Formen, Math. Ann. 88 (1923), 1-25.

3. J. Radon, Lineare Scharen orthogonalen Matrizen, Abh. Math. Sem. Univ. Hamburg 1 (1922), 1-14.

ManChester University, Manchester, England,

NEW YORK UNIVERSITY, AND

STANFORD UNIVERSITY 